\title{
Region Information-Based ROI Extraction by Multi-Initial Fast Marching Algorithm
}

\author{
Zhang Hongmei \\ School of Life Science and Technology, Xi'an Jiaotong University, Xi'an 710049, China \\ Email: claramei@mailst.xjtu.edu.cn \\ Bian Zhengzhong \\ School of Life Science and Technology, Xi'an Jiaotong University, Xi'an 710049, China \\ Email:bzzbme@mail.xjtu.edu.cn \\ Guo Youmin \\ First Affiliated Hospital, Xi'an Jiaotong University, Xi'an 710049, China \\ Email:ym.guo@china.com \\ Ye Min \\ Institute of Mechanical Engineering, Xi'an Jiaotong University, Xi'an 710064, China \\ Email: minye2000@263.net \\ Miao Yalin \\ School of Life Science and Technology, Xi'an Jiaotong University, Xi'an 710049, China \\ Email:myl@mailst.xjtu.edu.cn
}

Received 23 March 2003; Revised 10 January 2004; Recommended for Publication by Kyoung Mu Lee

\begin{abstract}
Region of interest (ROI) plays an important role in medical image analysis. In this paper, a new approach to ROI extraction based on the curve evolution is proposed. Different from the existent method, the proposed approach is efficient both in segmentation results and computational cost. The deforming curve is modeled as a monotonically marching front under a positive speed field, where a region speed function is derived by minimizing the new defined ROI energy, and integrated with the edge-based speed function. The curve evolution model integrating the ROI information has a large propagation range and could even drive the front in low-contrast and narrow thin areas. Moreover, a multi-initial fast marching algorithm, which permits the user to plant several seed curves as the initial front and evolves them simultaneously, is developed to fast implement the numerical solution. Selective planting seed curves could help the local growth and thus may further improve the segmentation results and reduce the computational cost. Experiments by our approach are presented and compared with that of the other methods, which show that the proposed approach could fast extract low-contrast and narrow thin ROI precisely.
\end{abstract}

Keywords and phrases: ROI extraction, curve evolution, multi-initial fast marching algorithm, front, segmentation.

\section{INTRODUCTION}

Region of interest (ROI) plays an important role in medical image analysis. Quantitative analysis of the shape and the properties of ROI could provide reliable data for diagnosing disease and the follow-up treatment planning [1]. As a result, to exploit accurate and fast ROI extraction method is in great need.

In recent years, ROI extraction based on the curve evolution approaches that deform an initial curve towards the desired boundary have been extensively exploited. Snakes or active contours first proposed by Kass et al. are energyminimizing curves that deform to fit the boundary of ROI [2]. The snakes are guided by the internal forces coming from the curve itself and external forces computed from the image data. Snakes and their variations are widely used in image segmentation. To overcome some drawbacks of classical snakes, region-based information are introduced to the model. Chakraborty et al. proposed the model that integrates the region-based segmentation and boundary finding in a unified framework [3]. In this approach, boundary is parameterized using Fourier descriptors which limit 
the shapes that they can describe. In addition, the distribution of the shape parameters is assumed to be multivariate Gaussian prior that may also present its limitations. The segmentation is formulated as maximum a posteriori probability, involving a lot of parameters to be estimated that bring great computational cost. Ivins and Zhu, respectively, proposed the statistical snakes for region growing and applied these models to image/texture segmentation $[4,5]$. However, due to its "Lagrangian" representation such that the coordinate system moves with the deforming curve, the parametrical snakes could not handle topological changes. To handle the splitting or the merging of the curve, extra reparameterization procedures must be performed during iteration, which brings expensive computational cost [6].

A major breakthrough is made by introducing the level set theory to curve propagation, resulting in a very elegant tool. The level set method proposed by Osher and Sethian offers a highly robust mathematic and numerical implementation on curve/surface evolution [7, 8]. Embedding the moving front to be zero level set of a higher dimensional function, topological changes can be handled naturally by exploiting the zero level set at any time. The level set method is introduced to image segmentation by Malladi et al. $[9,10,11]$. In this approach, selection of speed function is crucial. On one hand, the speed function controls the behavior of the front propagation; on the other hand, the form of the speed function decides the computational complexity of the numerical implementation. As a solution to the level set evolution equation, fast marching method may be the first choice for its cheap computational cost [10]. However, it could only be used for a monotonically marching front that requires the speed function to be always positive or negative. Narrowband method and Hermes algorithm can cope with all sorts of the level set evolution but their computational costs are still far more expensive than the fast marching method [12, 13]. Malladi et al. in [9] proposed the image-based positive speed function that could stop the front in the vicinity of the ROI boundary, but this only edge-based curve evolution may mislead the deformation at weak boundary, as the speed is too weak to propagate the front there. To address this problem, many improvements on designing the speed function are achieved by introducing region information to guide the curve deforming. Yezzi et al. proposed a fully global approach to image segmentation that is derived based on the deterministic principle of maximally separating the values of certain image statistics within a set of curves [14]. This is a pure region-based approach, thus it is very robust. However, because the image statistics are variable with the deforming curve, these statistics need to be estimated during the curve evolution, which may bring much computational cost. Moreover, it needs $n-1$ curves to segment $n$ regions with each curve corresponding to different curve evolution equation and level set function, which also present complex computation. Similar to Yezzi et al.'s work, nonparametric statistical method for image segmentation is proposed in [15], where the curve evolution aims at maximizing the mutual infor- mation within different curves. Another region-based curve evolution method is based on the criterion that the interior of the region has maximal similarity and different regions have maximal discrepancy [16]. But continually estimating variable statistics makes the computing expensive. Paragios and Deriche proposed the geodesic active regions by adding a region term onto the geodesic active contour model, which combines the region-based segmentation with edge information. The region term is derived as minimizing the negative log-likelihood function of the image, which is obtained by Markov random field (MRF) presegmentation [17]. Due to its complex form in speed function, the corresponding level set evolution equation is implemented by Hermes algorithm [13], which is more computationally expensive than the Fast Marching method.

Considering both the segmentation quality and the computational cost, in this paper, we propose an efficient approach to ROI extraction. Different from the other approaches, neither statistics are needed to be computed continuously nor complex numerical implementation is involved. The deforming curve is modeled as monotonically marching front under a new positive speed field, where a new region speed function is derived by minimizing the ROI energy. Integrating with the region information, the modified speed function has large propagation range and could even drive the front propagating in low-contrast and narrow thin areas. To further improve the segmentation results, multi-initial scheme is adopted [14] and the multiinitial fast marching algorithm is developed, which permits the user to plant several seed curves as the initial front and evolves them simultaneously. All the seed curves are treated as one complex front driven by the same evolution equation. Selective planting seed curves can avoid the monotonically marching front leaking out of the weak boundary too early to arrive at the desired boundary and it can also reduce the computational cost. Our approach is similar to that of Vilariño's cellular neural networks (CNN) approach to image segmentation $[18,19]$. Both approaches evolve pixel by pixel from their initial shapes and locations until delimiting the objects of interest, and the curve evolution is guided by local information from the image under consideration, which can offer a high flexible and efficient parallel processing.

The remainder of the paper is as follows: in Section 2, fast marching method is briefly outlined; in Section 3, the curve evolution model is proposed, where a new speed function is introduced by ROI energy minimizing; in Section 4, multi-initial fast marching algorithm is described in detail; in Section 5, experimental results are presented and compared with those of the other methods; finally in Section 6, conclusions are reported.

\section{FAST MARCHING METHOD}

Let $C(p, 0)$ be a closed parameterized curve in Euclidean plane $R^{2}$. Let $C(p, t)$ be the one-parameter family of curves generated by moving $C(p, 0)$ along its normal vector field $\vec{N}$ with speed $F$. The corresponding curve motion equation is 
given by

$$
\begin{gathered}
\frac{\partial C}{\partial t}=F \cdot \vec{N}, \\
C(p, 0)=C_{0}(p) .
\end{gathered}
$$

In particular, for the speed function $F$ is being always positive or negative, the front is marching monotonically. One way to characterize the position of this moving front is to compute the arrival time $T(x, y)$ of the front as it crosses each point $(x, y)$. By embedding the moving front to the level set of time function $T(x, y)$, thus the normal vector $\vec{N}$ could be give by $\vec{N}=\nabla T /|\nabla T|$, the fast marching equation is derived as follows [8]:

$$
\begin{aligned}
T(C(p, t)) \triangleq t & \Longrightarrow \nabla T \cdot C_{t}=1 \\
& \Longrightarrow \nabla T \cdot\left(F \cdot \frac{\nabla T}{|\nabla T|}\right)=1 \\
& \Longrightarrow F \cdot|\nabla T|=1 .
\end{aligned}
$$

The advantages of this equation representation are that it is intrinsic and that it is topologically flexible since at any time $t$, different topologies of $C$ can be handled naturally by exploiting the level set $\{C(p, t) \mid T(C(p, t))=t\}$.

\section{REGION-BASED CURVE EVOLUTION MODEL}

\subsection{ROI energy and region speed function}

Assume that ROI is the region enclosed by the moving front and is corresponding to the class $O$ in the image $I$. Let $\mu_{o}(I(x, y))$ denote the membership of the pixel belonging to the interesting object class $O$. Let

$$
P_{o}(I(x, y))= \begin{cases}1 & \text { if } \mu_{o}(I(x, y))>0.5 \\ -\varepsilon & \text { otherwise }\end{cases}
$$

where $\varepsilon \rightarrow 0^{+}$is a small positive constant. We define the ROI energy as follows:

$$
E_{\mathrm{ROI}}=-\iint_{\mathrm{ROI}} P_{O}(I(x, y)) d x d y .
$$

A direct explanation of (4) is that ROI should include as much pixels as possible belonging to the class $O$.

Using the Green theorem and variational method, we could derive the corresponding curve evolution equation as follows:

$$
\frac{\partial C}{\partial t}=-P_{O}(I) \cdot \vec{N}_{\text {in }}=P_{O}(I) \cdot \vec{N}
$$

where $\vec{N}$ is the outward normal vector. The detailed derivations are given in the appendix.

From (5), we could conclude that if a pixel belongs to the class $O$, this region force $P_{o}(I(x, y)) \cdot \vec{N}$ aims at expanding the front curve to include this pixel; otherwise, it aims at shrinking the front to exclude this pixel.
For many medical images, the gray values constitute an adequate statistic to distinguish one region from another. Therefore, histogram-based fuzzy cluster algorithm [20] is performed for initial segmentation to provide region information. Let $U=[u(l, k)](l=0, \ldots, 255 ; k=1, \ldots, K)$, where $u(l, k)$ denotes the membership of the gray level $l$ belonging to the $k$ th class and $K$ is the number of classes. Then $\mu_{o}(I)=u(I(\cdot, \cdot), O)$. The ROI class $O$ could be simply determined by mouse-choosing several pixels in this region.

\subsection{Modified speed function}

Malladi in $[9,10]$ proposes an image-based speed function:

$$
g_{I}=e^{-\alpha\left|\nabla G_{\sigma} * I\right|}, \quad \alpha>0,
$$

that could stop the front in the vicinity of the ROI boundary. However, this only edge-based speed is too weak to propagate the front in low-contrast and narrow thin areas. To address this problem, improvements have been exploited but that may bring expensive computational cost $[14,15,16,17]$. Considering both the segmentation quality and computational cost, we introduce the ROI information to the model by integrating the new region speed function $P_{O}(I)$ with the edge speed function $g_{I}$. The modified speed function is given by

$$
F_{\bmod i}=w_{R} \cdot P_{O}(I)+w_{E} \cdot g_{I}
$$

The corresponding curve evolution equation is

$$
\frac{\partial C}{\partial t}=F_{\bmod i} \cdot \vec{N}=w_{R} \cdot P_{O}(I) \cdot \vec{N}+w_{E} \cdot g_{I} \cdot \vec{N},
$$

where $w_{R}, w_{E} \in(0,1]$ are constants weighting the effects of region-based speed term and edge-based speed term, respectively.

If we choose $\varepsilon=\min \left\{w_{E} \cdot g_{I} / w_{R}\right\}$, the modified speed function $F_{\bmod i}=w_{R} \cdot P_{O}(I)+w_{E} \cdot g_{I}$ is always positive. The corresponding fast marching equation is given by

$$
F_{\bmod i} \cdot|\nabla T|=1 \text {. }
$$

The modified speed fuses both region and edge information that has large propagation range. Even at weak boundaries, it can provide proper speed to propagate the front.

\section{MULTI-INITIAL FAST MARCHING ALGORITHM}

Equation (8) can be implemented by the classical fast marching algorithm proposed by Malladi and Sethian [11]. However, monotonically marching front may leak out of the weak boundary too early to arrive at the desired ROI boundary. To address this problem, we develop the multi-initial fast marching algorithm that permits the user to plant seed curves as the initial front and evolves them simultaneously, which could perform the selective growth that may further improve the segmentation results and reduce the computational cost. 
(1) Initialization

Plant several seed curves in the ROI region;

Let initial front be the set of the pixels on all the seed curves;

Alive pixel.

The front pixels constitute the alive pixels. If we want the front to propagate outward, also tag as alive pixels in the interior of every seed curve; Assign alive pixels with zero crossing time $T_{\text {alive }}(i, j)=0$.

Trial pixel.

For each front pixel, the first-order neighborhood pixels are examined. If they are not labeled as alive, then, they become trial pixels with crossing time $T_{\text {trial }}(i, j)=1 / F_{\bmod i}(i, j)$.

Faraway pixel.

All other pixels are initialized as faraway with a

(2) Marching forward crossing time $T_{\text {faraway }}(i, j)=\infty$.

(If not satisfying stop criterion)

Let $A$ be the trial pixel with the smallest $T$ value.

Add the pixel $A$ to alive set and remove it from trial set.

Tag as trial all neighbors of $A$ that are not alive. If the neighbor is in faraway, remove, and add to the trial with initial crossing time $T(i, j)=1 / F_{\bmod i}(i, j)$.

Recompute the value of $T$ at all trial

End neighbors of $A$ according to (9).

Algorithm 1

The multi-initial fast marching algorithm is given in Algorithm 1.

An efficient technique of fast locating the grid pixel with smallest $T$ values in the narrowband is to use a variation on heapsort algorithm, resulting in only $O(N \log N)$ computational expense [10].

\section{EXPERIMENT}

To demonstrate the efficiency of our approach, the proposed curve evolution equation (8) for ROI extraction by multiinitial fast marching algorithm is compared with the other methods.

Method 1. ROI extraction based on the only edge-based curve evolution [9]:

$$
\frac{\partial C}{\partial t}=g \cdot \vec{N}
$$

Method 2. ROI extraction by geodesic active contours equation [21]:

$$
\frac{\partial C}{\partial t}=g\left(c_{1}+c_{2} \kappa\right) \cdot \vec{N}-(\nabla g \cdot \vec{N}) \cdot \vec{N} .
$$

Method 3. Geodesic active region equation proposed by Paragios and Deriche [17]:

$$
\begin{aligned}
\frac{\partial C}{\partial t}= & (1-\beta) \cdot\left[g\left(c_{1}+c_{2} \kappa\right) \cdot \vec{N}-(\nabla g \cdot \vec{N}) \cdot \vec{N}\right] \\
& +\beta \cdot \frac{\log P_{B}(I(x, y))}{\log P_{A}(I(x, y))} \cdot \vec{N} .
\end{aligned}
$$

Method 4. Fully global approach proposed by Yezzi et al. [14]:

$$
\frac{\partial C}{\partial t}=(u-v) \cdot\left(\frac{I-u}{A_{u}}+\frac{I-v}{A_{v}}\right) \cdot \vec{N}-c \cdot \kappa \cdot \vec{N} .
$$

$g$ is a monotonically decreasing function such that $g(r) \rightarrow 0$ as $r \rightarrow \infty$ and $g(0)=1$, and $\vec{N}$ is outward normal vector in (10) and (13) whereas inward normal vector in (11) and (12). $c_{1}, c_{2}, c$, and $\beta$ are constant. $P_{A}(I(x, y))$ and $P_{B}(I(x, y))$ are the joint probability of the image with respect to two class hypothesis $\mathrm{A}$ and $\mathrm{B}$, respectively. $u$ and $v$ are the average intensity inside and outside the deforming curve. The common choice of $g$ is given by (6). The numerical implementation of (8) and (10) is by our multi-initial fast marching algorithm. Due to the complex speed function form, the corresponding level set evolution equation of (11), (12), and (13) should be implemented by Hermes algorithm or by a more expensive narrowband method [12].

In the following experiments, we choose $\alpha=0.2, c_{1}=$ $-1, c_{2}=0.05, c=0.05$, and $\beta=0.7$. The ratio of $w_{R}$ and $w_{E}$ are recommended to be larger for low-contrast image or images with many narrow thin branches as the region information is more reliable than the edge information there. The ROI class $O$, the class numbers $K$ used in fuzzy cluster, and the values of $w_{E}$ and $w_{R}$ for each case are given in Table 1.

Figures 1, 2, 3, and 4 are the comparison results. The first column shows the initial seed curves, the second and the third column show the random middle state of the marching front, while the fourth column shows the final front state. Group (a) provide the results for our approach and group (b) show the results of Method 1, Method 2, Method 3, and Method 4, respectively.

Figure 1 is a sarcoma pathological brain MR image, where three tumors are to be extracted. In the initial state, three seed curves are planted in the tumor areas, therefore, tumors can be extracted at one time. It can be seen from Figure $1 \mathrm{~b}$ that because of the low-contrast and complex gradient of the ROI, the only edge-based speed misleads the deforming behavior. However, the results of Figure 1a are very promising, which show that the modified speed function integrating region information has large propagation range in low-contrast area.

Figure 2 is a meningioma pathological brain MR image, where the tumor area is the ROI. In this experiment, we want to compare the effect of the proposed region speed term with the advection speed term in geodesic active contours. In Figure 2a, the tumor extraction by the proposed curve evolution performs better in that it could even extract the narrow thin area on the top. Comparison of the results in Figure 2b shows that the geodesic active contour fails in extracting thin 
TABLE 1: Parameters for each case.

\begin{tabular}{c|ccccccccc}
\hline Figure number & 1 & 2 & 3 & 4 & 5 & 6 & 7 & 8 & 9 \\
\hline$w_{R}$ & 1.0 & 0.9 & 1.0 & 0.9 & Given & 1.0 & 0.9 & 0.9 & 1.0 \\
$w_{E}$ & 0.2 & 0.1 & 0.2 & 0.2 & Given & 0.2 & 0.2 & 0.2 & 0.2 \\
Interesting class & Three & Top & Right & DSA & Vessel & Vessel & Vessel & Vessel & Bone \\
$O$ & Tumors & Tumor & Tumor & Vessels & Branches & Branches & Branches & Branches & \\
$K$ & 3 & 5 & 5 & 2 & 2 & 2 & 2 & 2 & 2 \\
\hline
\end{tabular}
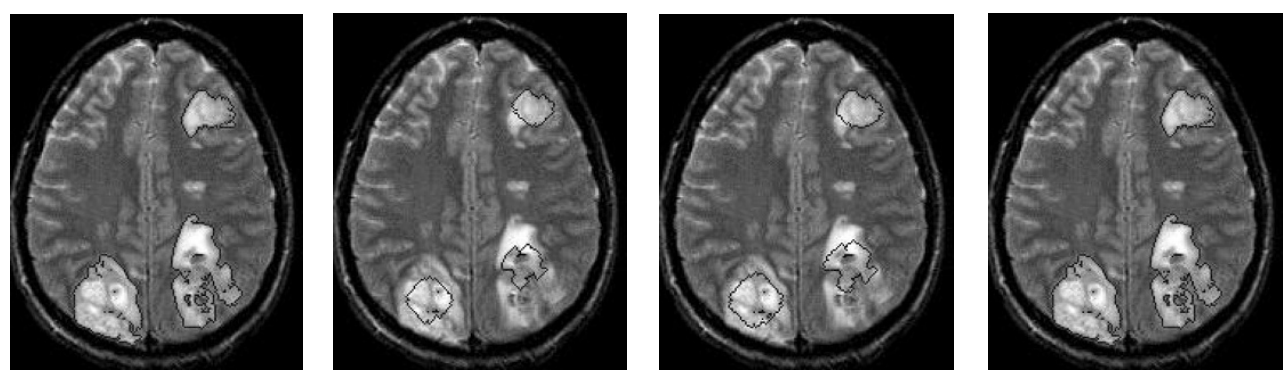

(a)
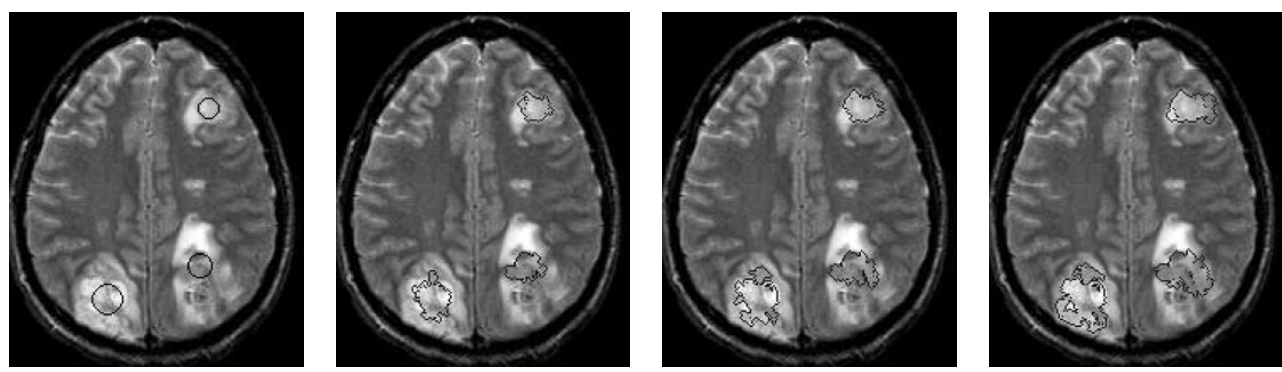

(b)

Figure 1: Sarcoma tumors extraction (a) by the proposed approach and (b) by Method 1.

area due to the low gradient information there. Experiments show that, compared with the advection term, the region force has larger attraction ability to guide the curve deforming in thin areas.

Figure 3 is a metastatic bronchogenic carcinoma pathological brain MR image, where the tumor on the right is the ROI. The result of our approach is slightly different from that of Paragios's geodesic active region method. The presegmentation map of Method 3 comes from MRF-based segmentation, and the region information used for curve evolution involves two terms: $P_{A}(I(x, y))$ and $P_{B}(I(x, y))$ that correspond to the joint probability of the image with respect to two-class hypothesis. However, our region information only involves the ROI class $O$ hypothesis. In addition, the computational cost of multi-initial fast marching algorithm used in our approach is $O(N \log N)$, whereas the Hermes algorithm used for Method 3 is much expensive.

Figure 4 is a DSA blood vessel. The result of our approach is almost the same as that of Method 4. However, the numerical implementation of Yezzi's equation needs to use narrowband algorithm that is far more expensive than fast marching algorithm. Moreover, the statistics of the average intensity $u$ and $v$ are variable with the deforming curve, thus continuously computing these statistics are needed during the curve evolution which brings much computational costs.

Considering both the computing cost and the segmentation results, our approach performs better than that of the other methods in that it runs faster and could locate the curve in the desired boundary as well, which is suitable for realtime medical image ROI extraction.

In our approach, choosing parameters of $w_{R}$ and $w_{E}$ is important to guide the curve deforming. Figure 5 show the impact of different combinations of $w_{R}$ and $w_{E}$ on the pulmonary vessels extraction. The obtained image is preprocessed by contrast enhancement, where vessels network is the ROI. From the comparison of the four groups in Figure 5, we can see that increasing the values for $w_{R}$ improves the image segmentation results significantly. Experimental results show that for low-contrast image or images with many narrow thin branches, larger $w_{R}$ could perform better as region information will play prominent role in guiding the front propagation there. 

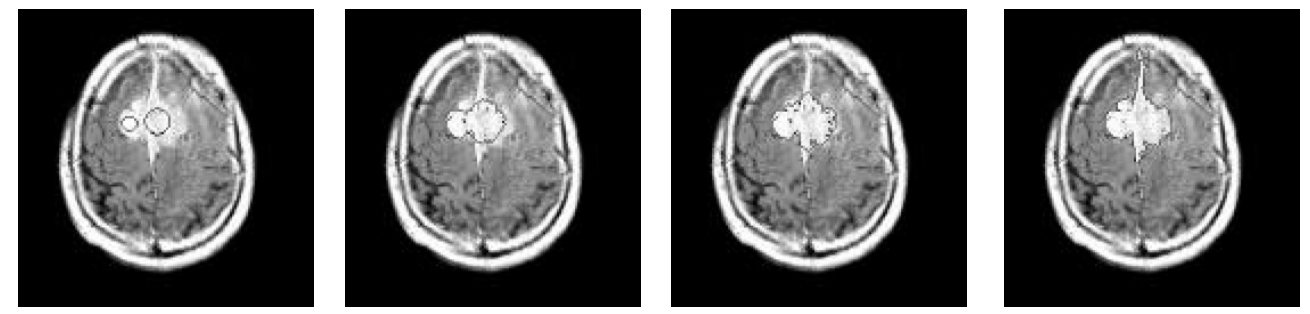

(a)
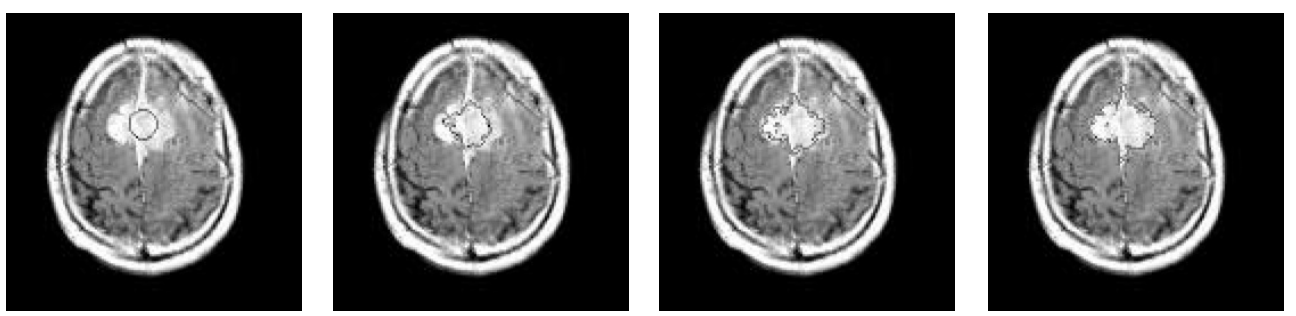

(b)

FIgURe 2: Meningioma tumor extraction (a) by the proposed approach and (b) by Method 2.
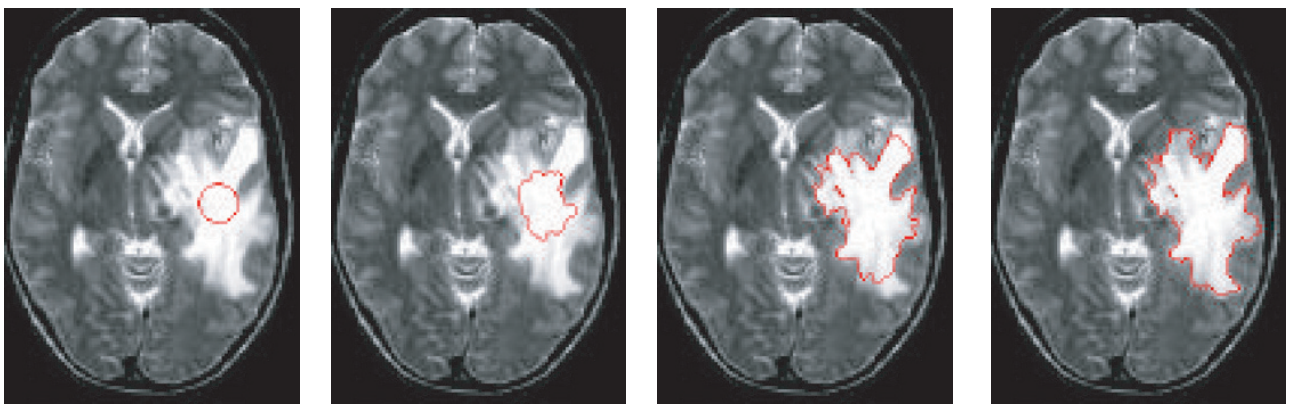

(a)
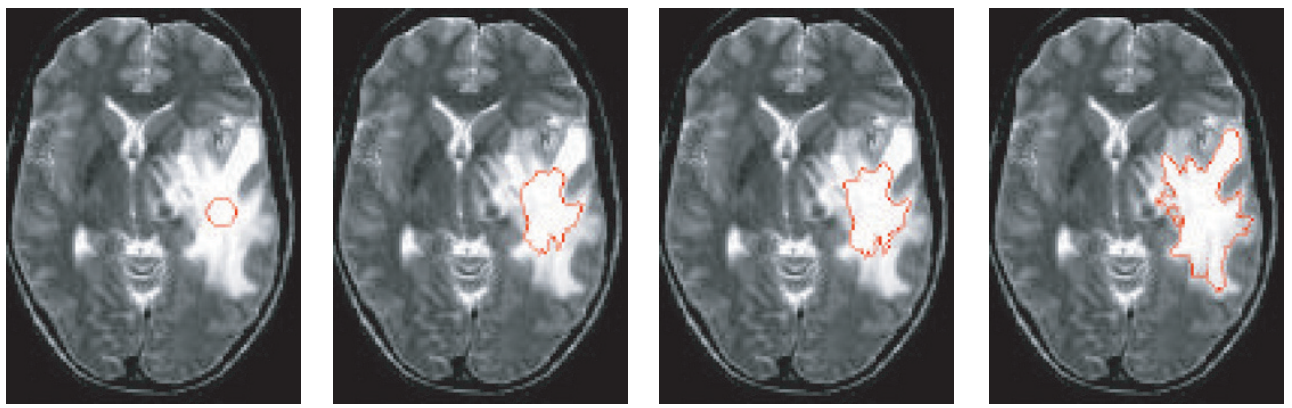

(b)

Figure 3: Carcinoma tumor extraction (a) by the proposed approach and (b) by Method 3.

In fast marching algorithm, because the front is monotonically marching, front leaking from boundary too early is a serious problem. Multi-initial planting seed curves can help in selective growth of the front that may avoid the early leaking problem. Seed curves are recommended to be planted in some narrow thin vessels branches or low-contrast area, in- ducing the front growth there. In addition, the interior of every seed curve is Alive pixels, which need not be updated in the marching processing, thus planting as much seed curves may reduce the computational cost. Figure 6 shows the results by our multi-initial fast marching algorithm and the classical fast marching algorithm without multi-initial. The 

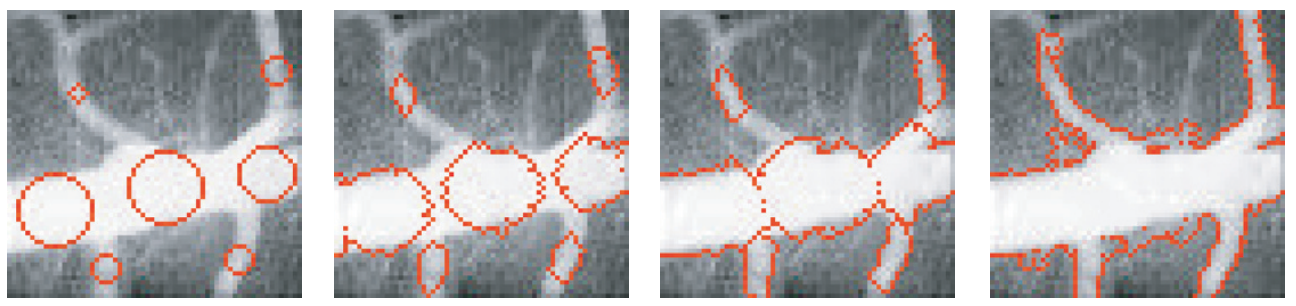

(a)
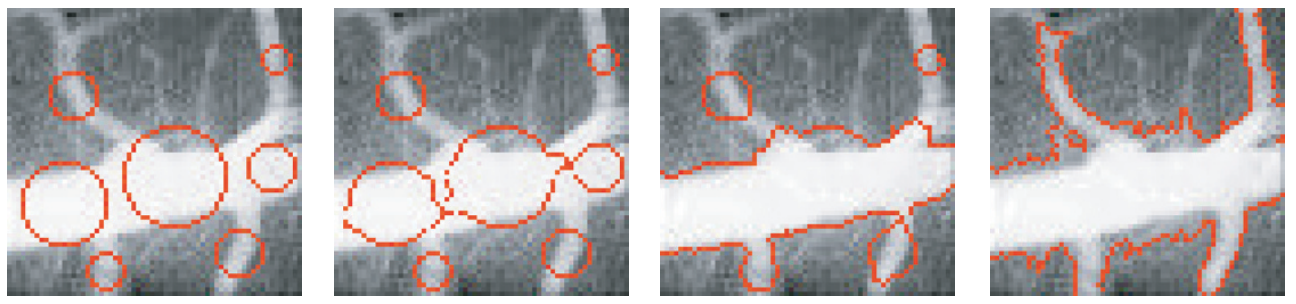

(b)

FIGURE 4: DSA vessel extraction (a) by the proposed approach and (b) by Method 4.

comparison show that selective planting of seed curves can do help in local growth of the front and also reduce the computational cost to some extent.

To further demonstrate the reliability of the proposed approach, experiments on several medical images segmentation are provided. Figures 7 and 8 are pulmonary vessels selected from CT images. Observation shows that the front stops at the desired vessel boundary encouragingly, and even some small and thin vessel branches, which exhibit much variability, could be located precisely as well. Almost the whole vessels network is extracted by our approach. Figure 9 is a bone image. From the segmentation results, we could see thin bones on the two sides that are precisely extracted by our approach.

\section{CONCLUSIONS}

In this paper, an efficient approach to ROI extraction based on the curve evolution was proposed. Region information was introduced to the model by minimizing the new defined ROI energy. Integrating region speed function with the edgebased speed term, the modified speed field has large propagation range even in low-contrast and narrow thin areas. Moreover, a multi-initial fast marching algorithm was developed, where selective planting seed curves may avoid the monotonically marching front leaking out of the weak boundary too early and further reduce the computational cost. ROI extraction on several medical images by our approach was provided and compared with that of the other methods. Experiments show that considering both the computational cost and segmentation results, the proposed curve evolution integrating region information could perform faster and could precisely locate the front at the desired boundary as well. Experimental results by our approach were very promising and it can be applied to medical image segmentation.
Nevertheless, due to the strict requirement of speed function form in the fast marching method, the proposed approach was only based on local image information, which involves a certain risk of being trapped in local minimum. Our future direction is to study global and real-time algorithm on clinical oriented medical image segmentation.

\section{APPENDIX}

Let

$$
E_{R}=\iint_{R} f(x, y) d x d y
$$

The goal is to find the boundary $\partial R$ of a region $R$ for a given function $f: R^{2} \rightarrow R$ that yields an extremum of the $E_{R}$; let

$$
\begin{aligned}
& Q=\frac{1}{2} \int_{0}^{x} f(t, y) d t, \\
& P=\frac{1}{2} \int_{0}^{y} f(x, t) d t .
\end{aligned}
$$

From Green theorem,

$$
\begin{aligned}
E_{R} & =\iint_{R}\left(\frac{\partial Q}{\partial x}+\frac{\partial P}{\partial y}\right) d x d y=\int_{\partial R}(Q d y-P d x) \\
& =\int_{\partial R}\left(Q \frac{d y}{d s}-P \frac{d x}{d s}\right) d s=\int_{0}^{L}\left(Q \frac{d y}{d s}-P \frac{d x}{d s}\right) d s \\
& \triangleq \int_{0}^{L} F\left[s, x(s), y(s), x^{\prime}(s), y^{\prime}(s)\right] d s
\end{aligned}
$$

where $s$ is the arc-length parameter and $L$ is the length of $\partial R$. From variational method, the corresponding Euler-lagrange 


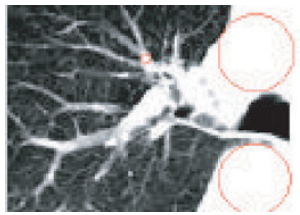

Initial state

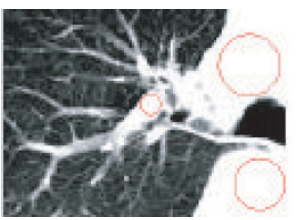

Initial state

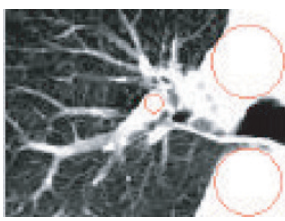

Initial state

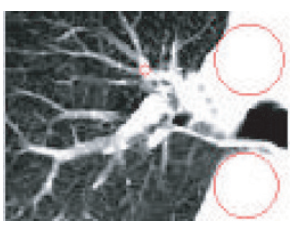

Initial state

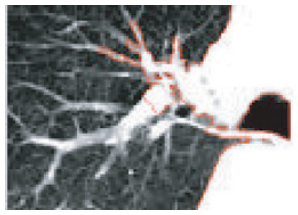

8280 iterations

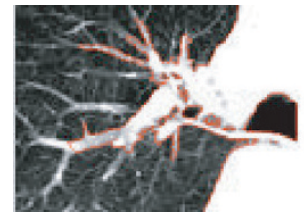

10440 iterations

(a)

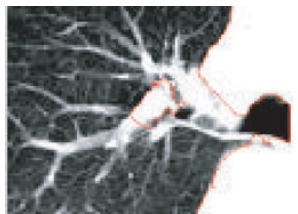

8280 iterations

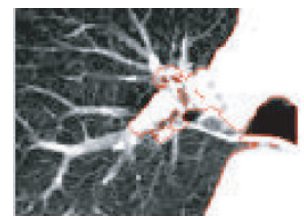

10440 iterations

(b)

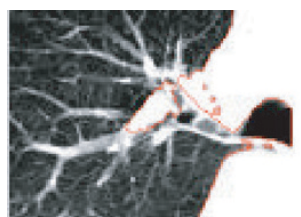

7560 iterations

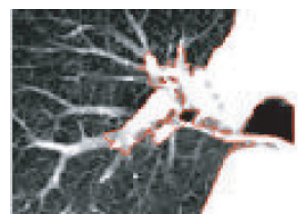

10080 iterations

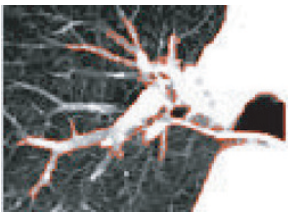

12180 iterations

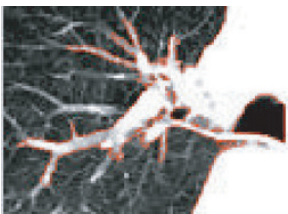

11100 iterations

(c)

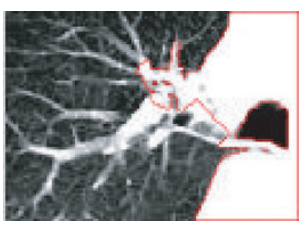

2520 iterations

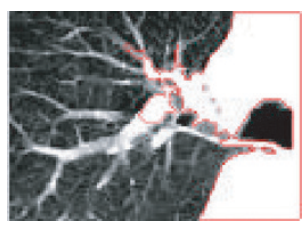

7560 iterations

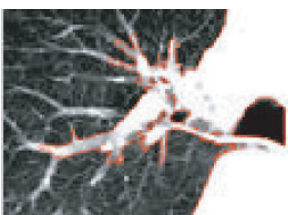

11580 iterations

(d)

FIGURE 5: Impact of different ratios of $w_{R}$ to $w_{E}$ on the pulmonary vessels extraction. (a) $w_{R}=0.9, w_{E}=0.2 ;$ (b) $w_{R}=0.6, w_{E}=0.4$; (c) $w_{R}=0.2, w_{E}=1.0 ;(\mathrm{d}) w_{R}=0, w_{E}=1.0$.

equation is

$$
\begin{aligned}
& F_{x}-\frac{d}{d s} F_{x^{\prime}}=0, \\
& F_{y}-\frac{d}{d s} F_{y^{\prime}}=0,
\end{aligned}
$$

which could yield the following equations:

$$
\begin{aligned}
& y^{\prime}(s) \frac{\partial Q}{\partial x}-x^{\prime}(s) \frac{\partial P}{\partial x}+\frac{d P}{d s}=0 \\
& y^{\prime}(s) \frac{\partial Q}{\partial y}-x^{\prime}(s) \frac{\partial P}{\partial y}-\frac{d Q}{d s}=0
\end{aligned}
$$

where

$$
\begin{aligned}
& \frac{d P}{d s}=x^{\prime}(s) \frac{\partial P}{\partial x}+y^{\prime}(s) \frac{\partial P}{\partial y} \\
& \frac{d Q}{d s}=x^{\prime}(s) \frac{\partial Q}{\partial x}+y^{\prime}(s) \frac{\partial Q}{\partial y} .
\end{aligned}
$$

Substituting (A.6) to (A.5), we could get

$$
\begin{gathered}
y^{\prime}(s) \cdot\left(\frac{\partial Q}{\partial x}+\frac{\partial P}{\partial y}\right)=0 \\
-x^{\prime}(s) \cdot\left(\frac{\partial P}{\partial y}+\frac{\partial Q}{\partial x}\right)=0 .
\end{gathered}
$$

Therefore,

$$
\begin{gathered}
y^{\prime}(s) \cdot f(x, y)=0, \\
-x^{\prime}(s) \cdot f(x, y)=0 .
\end{gathered}
$$

Using the gradient descent method, we could get the curve evolution equation:

$$
\begin{gathered}
\frac{\partial x}{\partial t}=-f(x, y) \cdot \frac{d y}{d s}, \\
\frac{\partial y}{\partial t}=f(x, y) \cdot \frac{d x}{d s} .
\end{gathered}
$$




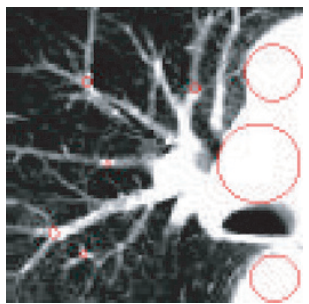

Initial state

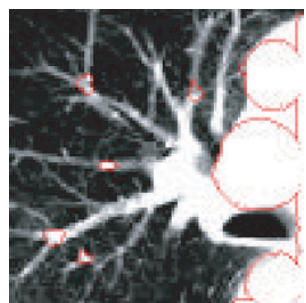

2160 iterations

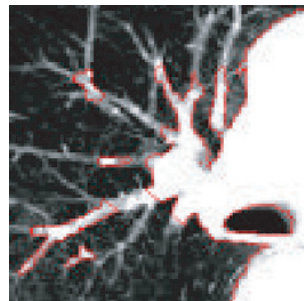

7200 iterations

(a)

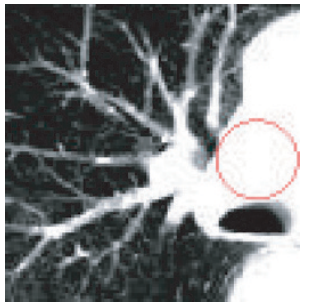

Initial state

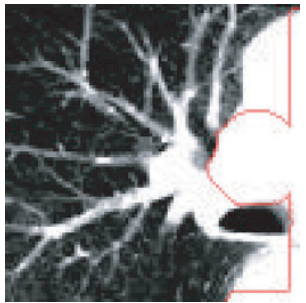

2160 iterations

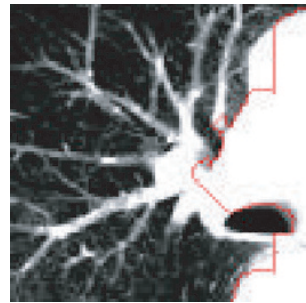

4680 iterations

(b)

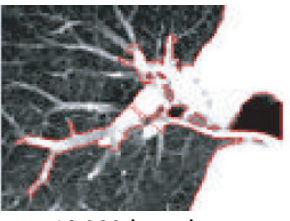

10080 iterations

(c)

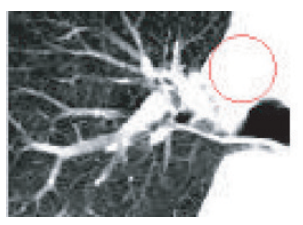

Initial state

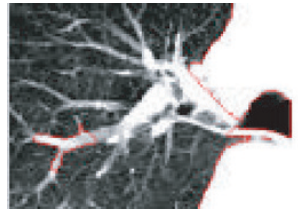

6120 iterations

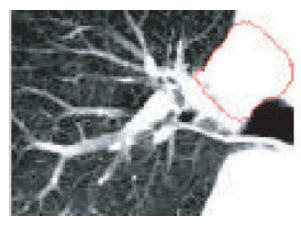

2340 iterations

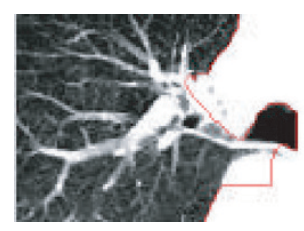

9180 iterations

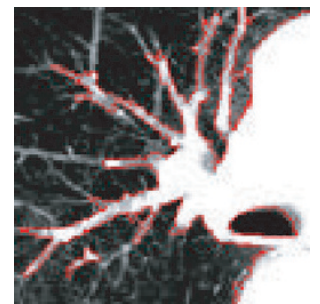

8940 iterations

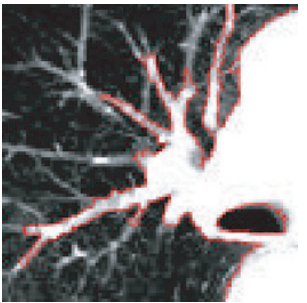

9480 iterations

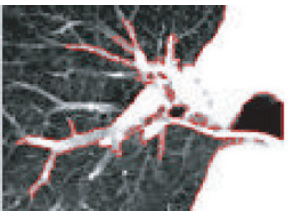

10860 iterations (d)

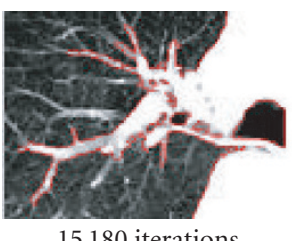

15180 iterations

FIGURE 6: Demonstration of selective planting seed curves: (a) and (b) demonstration of selective planting seed curves on helping local growth; (c) and (d) demonstration of selective planting seed curves on reducing computational cost.
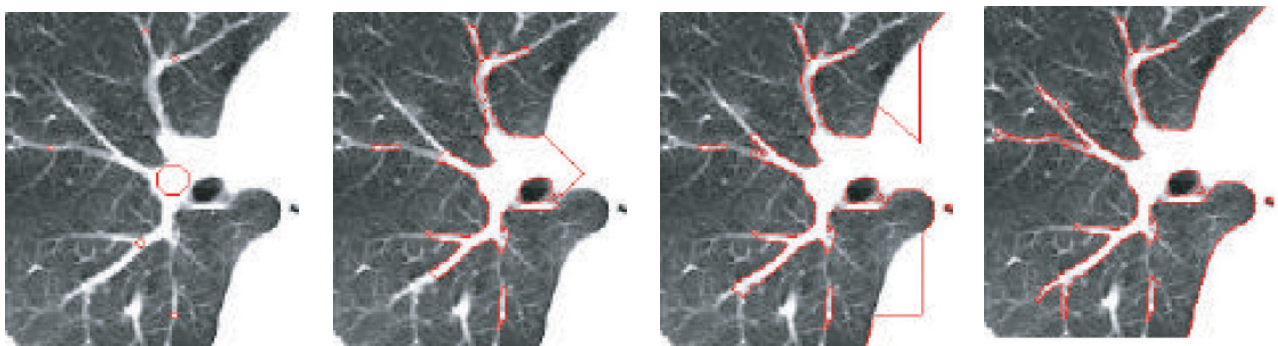

FIgURE 7: Pulmonary vessels extraction by the proposed approach. 

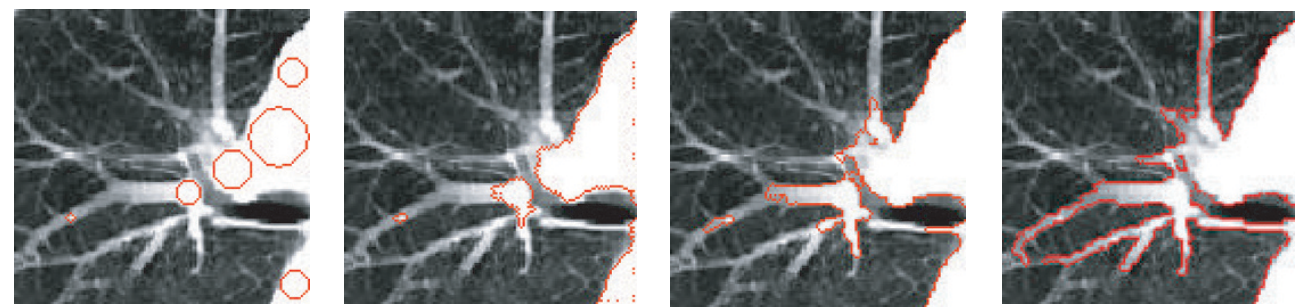

Figure 8: Pulmonary vessels extraction by the proposed approach.
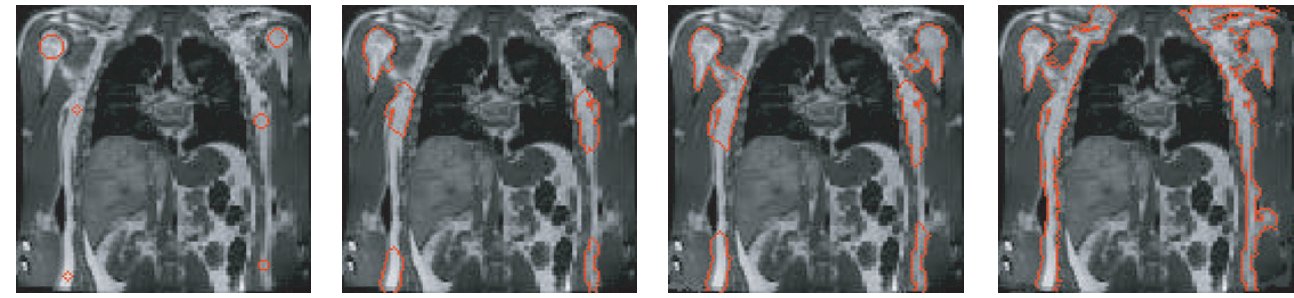

FIgURE 9: Medical bone image extraction by the proposed approach.

From differential geometry, we know that $\vec{\alpha}=(d x / d s, d y / d s)$ is the unit tangent vector, so $\vec{N}=(-d y / d s, d x / d s)$ is the unit inward normal vector. Thus we obtain

$$
\frac{\partial C}{\partial t}=f(x, y) \cdot \vec{N}
$$

\section{REFERENCES}

[1] L. M. Lorigo, O. D. Faugeras, W. E. L. Grimson, et al., "CURVES: curve evolution for vessel segmentation," Medical Image Analysis, vol. 5, no. 3, pp. 195-206, 2001.

[2] M. Kass, A. Witkin, and D. Terzopoulos, "Snakes: active contour models," in Proc. 1st International Conference on Computer Vision (ICCV'87), pp. 259-268, London, UK, June 1987.

[3] A. Chakraborty, L. H. Staib, and J. S. Duncan, "Deformable boundary finding in medical images by integrating gradient and region information," IEEE Trans. on Medical Imaging, vol. 15, no. 6, pp. 859-870, 1996.

[4] J. Ivins and J. Porrill, "Active region models for segmenting textures and colours," Image and Vision Computing, vol. 13, no. 5, pp. 431-438, 1995.

[5] S. C. Zhu and A. Yuille, "Region competition: unifying snakes, region growing, and Bayes/MDL for multiband image segmentation," IEEE Trans. on Pattern Analysis and Machine Intelligence, vol. 18, no. 9, pp. 884-900, 1996.

[6] T. McInerney and D. Terzopoulos, "T-snakes: topology adaptive snakes," Medical Image Analysis, vol. 4, no. 2, pp. 73-91, 2000.

[7] S. Osher and J. A. Sethian, "Fronts propagating with curvature dependent speed: algorithms based on Hamilton-Jacobi formulations," Journal of Computational Physics, vol. 79, no. 1, pp. 12-49, 1988.

[8] J. A. Sethian, Level Set Methods and Fast Marching Methods: Evolving Interfaces in Computational Geometry, Fluid Mechanics, Computer Vision, and Materials Science, Cambridge University Press, New York, NY, USA, 1999.

[9] R. Malladi, J. A. Sethian, and B. C. Vemuri, "Shape modeling with front propagation: a level set approach," IEEE Trans. on
Pattern Analysis and Machine Intelligence, vol. 17, no. 2, pp. 158-175, 1995.

[10] R. Malladi and J. A. Sethian, "An $O(N \log N)$ algorithm for shape modeling," Proceedings of the National Academy of Sciences, vol. 93, no. 18, pp. 9389-9392, 1996.

[11] R. Malladi and J. A. Sethian, "A real-time algorithm for medical shape recovery," in Proc. 6th IEEE International Conference on Computer Vision (ICCV'98), pp. 304-310, Bombay, India, January 1998.

[12] D. Adalsteinsson and J. Sethian, "A fast level set method for propagating interfaces," Journal of Computational Physics, vol. 118, no. 2, pp. 269-277, 1995.

[13] N. Paragios and R. Deriche, "A PDE-based level-set approach for detection and tracking of moving objects," in Proc. 6th IEEE International Conference on Computer Vision (ICCV'98), pp. 1139-1145, Bombay, India, January 1998.

[14] A. Yezzi Jr., A. Tsai, and A. Willsky, "A fully global approach to image segmentation via coupled curve evolution equations," Journal of Visual Communication and Image Representation, vol. 13, no. 1/2, pp. 195-216, 2002.

[15] J. Kim, J. W. Fisher, A. Yezzi Jr., M. Cetin, and A. S. Willsky, "Nonparametric methods for image segmentation using information theory and curve evolution," in Proc. IEEE International Conference on Image Processing (ICIP '02), vol. 3, pp. 797-800, Rochester, NY, USA, June 2002.

[16] B. Sumengen, B. S. Manjunath, and C. Kenney, "Image segmentation using curve evolution and region stability," in Proc. 16th IEEE International Conference on Pattern Recognition (ICPR '02), vol. 2, pp. 965-968, Quebec City, Canada, August 2002.

[17] N. Paragios and R. Deriche, "Geodesic active regions for motion estimation and tracking," in Proc. 7th IEEE International Conference on Computer Vision (ICCV '99), vol. 1, pp. 688694, Kerkyra, Greece, September 1999.

[18] D. L. Vilariño, V. M. Brea, D. Cabello, and J. M. Pardo, "Discrete-time CNN for image segmentation by active contours," Pattern Recognition Letters, vol. 19, no. 8, pp. 721-734, 1998.

[19] D. L. Vilariño, D. Cabello, X. M. Pardo, and V. M. Brea, "Cellular neural networks and active contours: a tool for image 
segmentation," Image and Vision Computing, vol. 21, no. 2, pp. 189-204, 2003.

[20] J. C. Bezdek, Pattern Recognition with Fuzzy Objective Function Algorithms, Plenum Press, New York, NY, USA, 1981.

[21] V. Caselles, R. Kimmel, and G. Sapiro, "Geodesic active contours," International Journal of Computer Vision, vol. 22, no. 1, pp. 61-79, 1997.

Zhang Hongmei was born in 1973. She is currently a Ph.D. candidate at the School of Life Science and Technology, Xi' an Jiaotong University, China. She received her B.S. degree in material science from Nanjing University of Science and Technology in 1996, and her M.S. degree from Xi'an Jiaotong University in the area of computing mechanics in 2000. Her research interests are in the theory and technology of medical image segmentation and fusion, machine learning, and bioinformatics.

Bian Zhengzhong was born in 1938. He is currently a Professor and Doctor Supervisor at the School of Life Science and Technology, Xi'an Jiaotong University, China. He received his B.S. degree in wireless engineering from Xi'an Jiaotong University in 1961. From 1982 to 1984, he studied at Oakland University as a Visiting Scholar. His research interests are in medical image processing and imaging, development of medical instrument, and bioinformatics.

Guo Youmin was born in 1955. He is currently a Professor and Doctor Supervisor at the Department of Radiology in the First Affiliated Hospital, Xi'an Jiaotong University, China. He received his B.S., M.S., and Ph.D. degrees in medical imaging and radiology from Xi'an Medical University in 1979, 1994, and 1999, respectively. His research interests are in radiological diagnosis on chest.

Ye Min was born in 1978. He is currently a Ph.D. candidate at the School of Mechanical Engineering, Xi'an Jiaotong University, China. He received his B.S. and M.S. degrees in mechanical engineering from Chang'an University in 2000 and 2003, respectively. His research interests are in pattern recognition and fuzzy control.
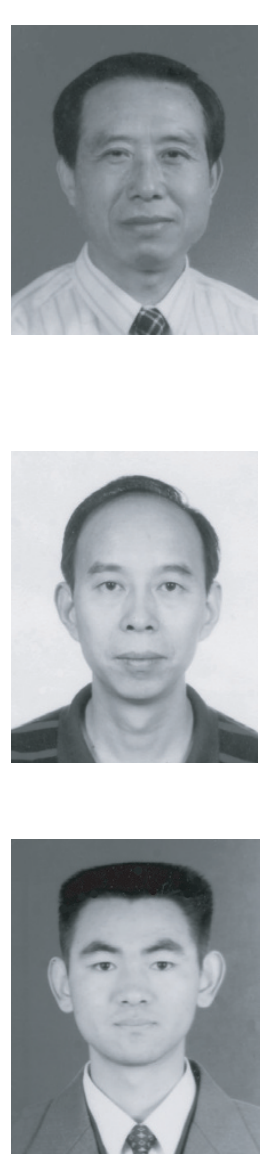

Miao Yalin was born in 1974 . He is currently a Ph.D. candidate at the School of Life Science and Technology, Xi' an Jiaotong University, China. He received his B.S. degree in energy and power engineering and his M.S. degree in computer science from Xi'an Jiaotong University in 1994 and 2002, respectively. His research interests are in medical image processing and bioinformat-
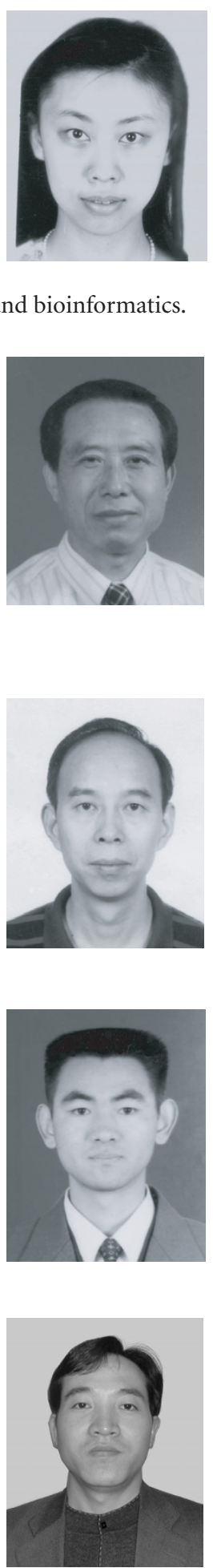
ics. 